EPJ Web of Conferences 72, 00011 (2014)

DOI: 10.1051/epjconf / 20147200011

(C) Owned by the authors, published by EDP Sciences, 2014

\title{
Recent results from BESIII experiment
}

\author{
Hai-Bo Li ${ }^{1, a}$ (On Behalf of the BESIII Collaboration) \\ ${ }^{1}$ Institute of High Energy Physics, Chinese Academy of Sciences, Beijing 100049, China
}

\begin{abstract}
In this talk, we present recent results from BESIII experiment. This talk covers the studies of charmonium(-like) states, light hadron spectroscopy and charm physics at the BESIII.
\end{abstract}

\section{Introduction}

The Beijing Electron Collider has been upgraded to a double ring collider with a design luminosity of $1 \times$ $10^{33} \mathrm{~cm}^{-2} \mathrm{~s}^{-1}$ at a center-of-mass energy of $3.78 \mathrm{GeV} / \mathrm{c}^{2}$. It is operating between 2.0 and $4.6 \mathrm{GeV} / \mathrm{c}^{2}$ in the center of mass energy. The BESIII experiment is used to study the charm and $\tau$ physics. The BESIII experiment at the BEPCII collider started data taking since 2008, and data samples were accumulated at the peak of the narrow vector charmonium resonances as well as above $4.0 \mathrm{GeV} / \mathrm{c}^{2}$. Samples of 13 billion $\mathrm{J} / \psi$ decay events and 0.5 billion $\psi(2 S)$ decay events have been collected for the study of charmonium decays and light hadron spectroscopy in the charmonium decays. For the study of charm meson decays, the BESIII experiment acquired about $2.9 \mathrm{fb}^{-1}$ on the $\psi(3770)$ peak. Between December of 2012 and June of 2013, the BESIII detector collected a total integrated luminosity of $2.9 \mathrm{fb}^{-1}$ of data samples, especially, the largest samples collected at $E_{C M}=4.23 \mathrm{GeV} / \mathrm{c}^{2}\left(1054 \mathrm{pb}^{-1}\right)$, $4.26 \mathrm{GeV} / \mathrm{c}^{2}\left(806 \mathrm{pb}^{-1}\right)$, and $4.36 \mathrm{GeV} / \mathrm{c}^{2}\left(523 \mathrm{pb}^{-1}\right)$, these data samples have provided powerful study of the $X Y Z$ states.

In this talk, we present the most recent results on the studies of the charged charmonium-like states, charmonium decays, light hadron spectroscopy and charm physics at the BESIII experiment.

\section{Observations of the charmonium-like states}

Many charmonium-like states, " $X Y Z$ " particles, have been observed in the initial state radiation (ISR) process and $B$ meson decays. All these states populate the charmonium mass region above open charm threshold. Many strange properties measured from these states make them more like exotic states rather than conventional mesons. The BESIII experiment is unique to use $e^{+} e^{-}$collision tuned to center of mass energies to directly produce the vector

\footnotetext{
ae-mail: lihb@ihep.ac.cn
}

charmonium-like states, such as $Y(4260)$ or $Y(4360)$, respectively. These data samples can be used not only to study the $Y(4260)$ or $Y(4360)$ states, but also to search for the "charged charmonium-like states", $Z_{c}(3900), Z_{c}(4020)$ and $Z_{c}(4025)$.

\subsection{The charged $Z_{c}(3900)$ in $e^{+} e^{-} \rightarrow \pi^{+} \pi^{-} J / \psi$}

The BESIII experiment studied the process $e^{+} e^{-} \rightarrow$ $\pi^{+} \pi^{-} J / \psi$ at a center-of-mass (CM) energy of $4.26 \mathrm{GeV} / \mathrm{c}^{2}$ using $525 \mathrm{pb}^{-1}$ data sample [1]. A structure at around 3.9 $\mathrm{GeV} / \mathrm{c}^{2}$ is observed in the $\pi^{ \pm} J / \psi$ mass spectrum, which is referred to as the $Z_{c}$ (2900). This structure couples to charmonium and has an electric charge, which is suggestive of a state containing more quarks than just a charm and anti-charm. A fit to the $\pi^{ \pm} J / \psi$ invariant mass spectrum as shown in Fig. 1 (Top), neglecting interference, results in a mass of $(3899.0 \pm 3.6 \pm 4.9) \mathrm{MeV} / \mathrm{c}^{2}$ and a width of $(46 \pm 10 \pm 20) \mathrm{MeV}$ [1]. This structure, was also observed at the same time by the Belle collaboration [2] and was confirmed by the authors of Ref. [3] using CLEO-c data. The cross section of $e^{+} e^{-} \rightarrow \pi^{+} \pi^{-} J / \psi$ is measured to be $(62.9 \pm 1.9 \pm 3.7) \mathrm{pb}$ at the $\mathrm{CM}$ energy of $4.26 \mathrm{GeV} / \mathrm{c}^{2}$, which agrees with the existing results from the BaBar, Belle, and CLEO experiments. The $Z_{c}(3900)$ production ratio is also measured to be $R=\frac{\sigma\left(e^{+} e^{-} \rightarrow \pi^{ \pm} Z_{c}(3900)^{\mp}\right)}{\sigma\left(e^{+} e^{-} \rightarrow \pi^{+} \pi^{-} J / \psi\right)}=$ $(21.5 \pm 3.3 \pm 7.5) \%[1]$.

\subsection{Structure in charged $D^{*} \bar{D}+$ c.c.}

One possible clue for the interpretation of the $Z_{c}(3900)$ is that it has a mass very close to $D^{*} \bar{D}$ threshold. To investigate, the BESIII experiment studied the processes $e^{+} e^{-} \rightarrow \pi^{-} D^{+} \bar{D}^{* 0}+$ c.c. and $e^{+} e^{-} \rightarrow \pi^{-} \bar{D} D^{*+}+$ c.c. at the $\mathrm{CM}$ of $4.26 \mathrm{GeV} / \mathrm{c}^{2}$ [4]. Clear structure in the mass spectrum of both $D^{+} \bar{D}^{* 0}+$ c.c. and $\bar{D} D^{*+}+$ c.c. was found as shown in Fig. 1 (Bottom). The measured mass and width were $(3883.9 \pm 1.5 \pm 4.2) \mathrm{MeV} / \mathrm{c}^{2}$ and $(24.8 \pm 3.3 \pm 11.0) \mathrm{MeV} / \mathrm{c}^{2}$, respectively. The structure was named as $Z_{c}(3885)$, and its mass and width are both slightly lower than the ones of $Z_{c}(3900)$ in the $\pi^{ \pm} J / \psi$, but 

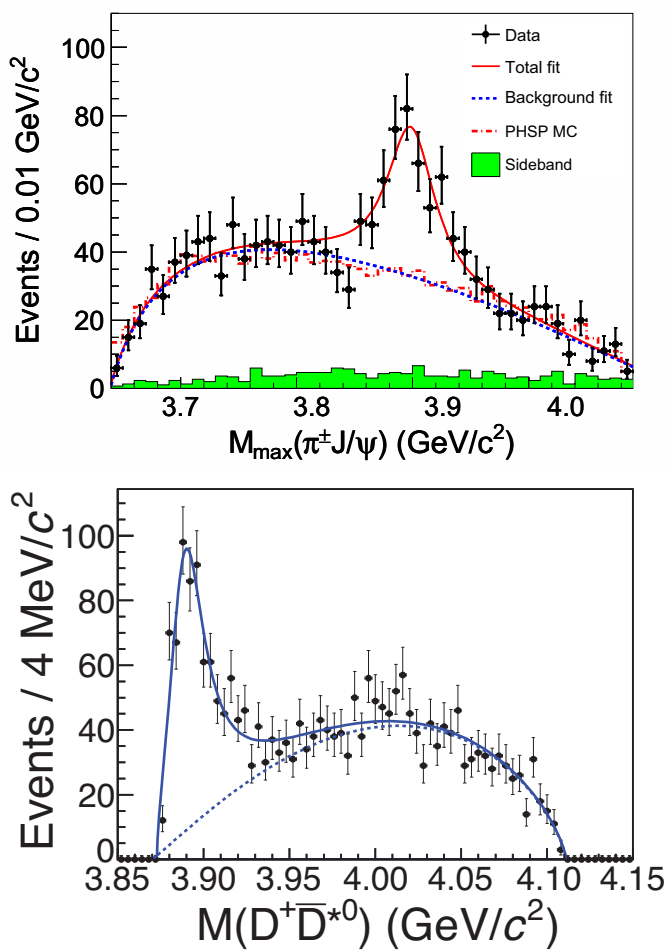

Figure 1. Top: The $Z_{c}(3900)$ produced in the $e^{+} e^{-} \rightarrow \pi^{ \pm} J / \psi$ at $\mathrm{CM}$ of $4.26 \mathrm{GeV} / \mathrm{c}^{2}$ and decaying through $Z_{c}(3900)^{ \pm} \rightarrow$ $\pi^{ \pm} J / \psi$ [1]. Bottom: Structure in $D^{+} \bar{D}^{* 0}+$ c.c. from the reaction $e^{+} e^{-} \rightarrow \pi^{-} D^{+} \bar{D}^{* 0}+$ c.c. at $\mathrm{CM}$ of $4.26 \mathrm{GeV} / \mathrm{c}^{2}$ [4].

both structures are likely related. The angular distribution of the $\pi Z_{c}(3885)$ system favors a $J^{P}=1^{+}$quantum number assignment for the structure and disfavors $1^{-}$or $0^{-}$. The Born cross section times the $D \bar{D}^{*}$ branching fraction of the $Z_{c}(3885)$ is measured to be $\sigma\left(e^{+} e^{-} \rightarrow \pi^{ \pm} Z_{c}(3885)^{\mp}\right) \times$ $\mathcal{B}\left(Z_{c}(3885)^{\mp} \rightarrow\left(D \bar{D}^{*}\right)^{\mp}\right)=(83.5 \pm 6.6 \pm 22.0) \mathrm{pb}$. Assuming the $Z_{c}(3885) \rightarrow D \bar{D}^{*}$ signal reported here and the $Z_{c}(3900) \rightarrow \pi J / \psi$ signal are from the same source, the partial width ratio $\frac{\Gamma\left(\mathcal{Z}_{c}(3885) \rightarrow D \bar{D}^{*}\right)}{\Gamma\left(\mathcal{Z}_{c}(3900) \rightarrow \pi J / \psi\right)}=6.2 \pm 1.1 \pm 2.7$ is determined [4].

\subsection{Charge structures in $\left(D^{*} \bar{D}^{*}\right)^{ \pm}$and $\pi^{ \pm} h_{c}$}

BESIII studied the process $e^{+} e^{-} \rightarrow\left(D^{*} \bar{D}^{*}\right)^{ \pm} \pi^{\mp}$ at a $\mathrm{CM}$ energy of $4.26 \mathrm{GeV} / \mathrm{c}^{2}$ using a $827 \mathrm{pb}^{-1}$ data sample [5]. Based on a partial reconstruction technique, the Born cross section is measured to be $(137 \pm 9 \pm 15) \mathrm{pb}$. A structure near the $\left(D^{*} \bar{D}^{*}\right)^{ \pm}$threshold in the $\pi^{\mp}$ recoil mass spectrum is observed (see Fig. 2 (Top)), which is denoted as the $Z_{c}^{ \pm}(4025)$ [5]. The measured mass and width of the structure are $(4026.3 \pm 2.6 \pm 3.7) \mathrm{MeV} / \mathrm{c}^{2}$ and $(24.8 \pm 5.6 \pm 7.7) \mathrm{MeV} / \mathrm{c}^{2}$, respectively. Its production ratio $\frac{\sigma\left(e^{+} e^{-} \rightarrow Z_{c}^{ \pm}(4025) \pi^{\mp} \rightarrow\left(D^{*} D^{*}\right)^{\mp} \pi^{\mp}\right)}{\sigma\left(e^{+} e^{-} \rightarrow\left(D^{*} D^{*}\right)^{ \pm} \pi^{\mp}\right)}$ is determined to be $0.65 \pm 0.09 \pm 0.06$. Since this structure couples to $\left(D^{*} \bar{D}^{*}\right)^{ \pm}$ and has electric charge, the observation suggests that the $Z_{c}(4025)$ may be a loosely bound $\left(D^{*} \bar{D}^{*}\right)^{ \pm}$system [6].

The BESIII experiment also studied $e^{+} e^{-} \rightarrow \pi^{+} \pi^{-} h_{c}$ at $\mathrm{CM}$ energies from $3.90 \mathrm{GeV} / \mathrm{c}^{2}$ to $4.42 \mathrm{GeV} / \mathrm{c}^{2}$ [7]. The
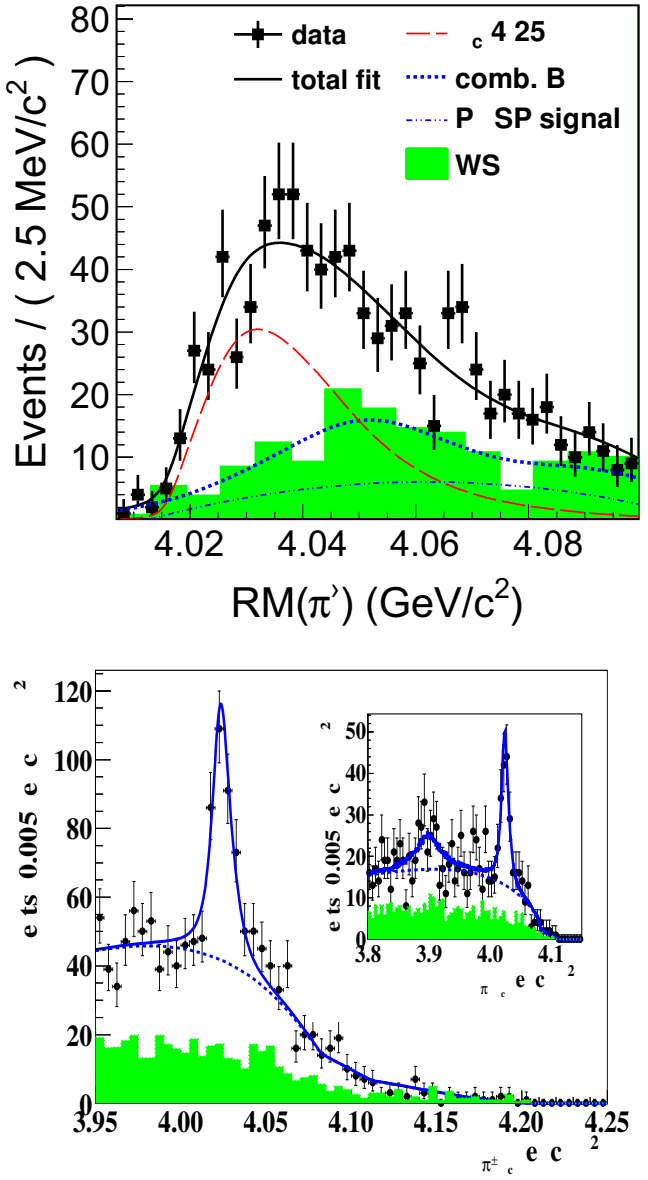

Figure 2. Top: Structure in the $\left(D^{*} \bar{D}^{*}\right)^{ \pm}$from the reaction $e^{+} e^{-} \rightarrow \pi^{\mp}\left(D^{*} \bar{D}^{*}\right)^{ \pm}$at $\mathrm{CM}$ energy of $4.26 \mathrm{GeV} / \mathrm{c}^{2}$ [5]. Bottom: the $Z_{c}(4020)$ produced in the $e^{+} e^{-} \rightarrow \pi^{\mp} Z_{c}(4020)^{ \pm}$at $\mathrm{CM}$ energies of $4.23,4.26$ and $4.36 \mathrm{GeV} / \mathrm{c}^{2}$ and decaying through $Z_{c}(4020) \rightarrow \pi^{ \pm} h_{c}[7]$.

Born cross sections are measured at 13 energies, and are found to be of the same order of magnitude as those of $e^{+} e^{-} \rightarrow \pi^{+} \pi^{-} J / \psi$ but with a different line shape. In the $\pi^{ \pm} h_{c}$ mass spectrum, a distinct structure, referred to as $Z_{c}(4020)$, is observed at $4.02 \mathrm{GeV} / c^{2}$. The $Z_{c}(4020)$ carries an electric charge and couples to charmonium, which is suggestive of a state containing more quarks than just a charm and an anti-charm quark, as the $Z_{c}(3900)$ observed in the $\pi^{ \pm} J / \psi$ system. A fit to the $\pi^{ \pm} h_{c}$ invariant mass spectrum (see Fig. 2 (Bottom)), neglecting possible interferences, results in a mass of $(4022.9 \pm 0.8 \pm 2.7) \mathrm{MeV} / c^{2}$ and a width of $(7.9 \pm 2.7 \pm 2.6) \mathrm{MeV} / \mathrm{c}^{2}$ for the $Z_{c}(4020)$, where the first errors are statistical and the second systematic. The difference between the parameters of this structure and the $Z_{c}(4025)$ observed in $D^{*} \bar{D}^{*}$ final state [5] is within $1.5 \sigma$, but whether they are the same state needs further investigation. No significant $Z_{c}(3900)$ signal is observed, and upper limit on the $Z_{c}(3900)$ production cross section in $\pi^{ \pm} h_{c}$ at $\mathrm{CM}$ energy of $4.26 \mathrm{GeV} / \mathrm{c}^{2}$ is set to be smaller than $11 \mathrm{pb}$ at the $90 \%$ C.L., which is lower than that of $e^{+} e^{-} \rightarrow \pi^{ \pm} Z_{c}(3900)^{\mp}, Z_{c}(3900) \rightarrow \pi^{\mp} J / \psi[1]$. 

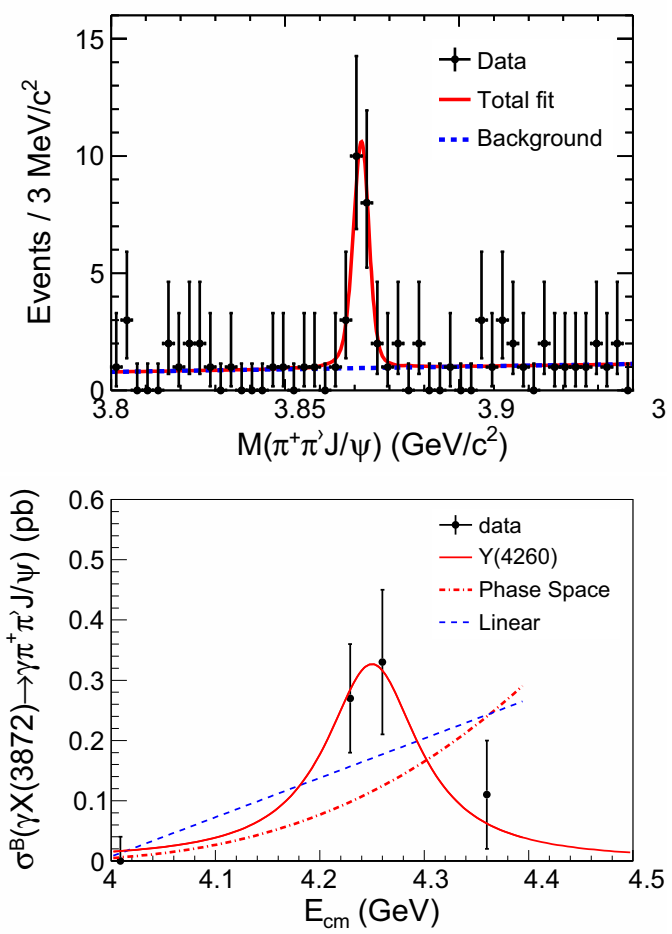

Figure 3. Top: the $X$ (3872) produced in $e^{+} e^{-} \rightarrow \gamma X(3872)$ at $E_{C M}=4.23,4.26$, and $4.36 \mathrm{GeV} / \mathrm{c}^{2}$ and decaying through $X(3872) \rightarrow \pi^{+} \pi^{-} J / \psi$. Bottom: The cross section for $e^{+} e^{-} \rightarrow$ $\gamma X(3872)$. The cross section is more consistent with a $Y(4260)$ shape than phase space or a linear lineshape.

\subsection{Observation of the $X(3872)$}

The $X(3872)$ was observed by Belle in $B^{ \pm} \rightarrow K^{ \pm} \pi^{+} \pi^{-} J / \psi$ decays ten years ago [8]. It was confirmed subsequently by several other experiments [9-11]. Since its discovery, it triggered many speculations about its nature, including a hadronic molecule or teraquark state. The CDF and $\mathrm{LHCb}$ experiments determined the spin-parity of the $X(3872)$ being $J^{P}=1^{+}[12,13]$, and $\mathrm{CDF}$ experiment also found that the $\pi^{+} \pi^{-}$system was dominated by a $\rho(770)$ resonance [14]. Using data samples at center-of-mass energies from 4.009 to $4.420 \mathrm{GeV} / \mathrm{c}^{2}$, the $e^{+} e^{-} \rightarrow \gamma X(3872)$ was observed for the first time [15]. The $X(3872)$ is clearly seen in the $M\left(\pi^{+} \pi^{-} J / \psi\right)$ (summed over all energy points) displayed in Fig. 3 (Top) . The measured mass, $M=3871.9 \pm 0.7 \pm 0.2 \mathrm{MeV} / \mathrm{c}^{2}$, is consistent with previous measurements. As shown in Fig. 3 (Bottom), the cross section of this process as a function of center of mass energy, while not conclusive, suggests this observation could arise from the process $Y(4260) \rightarrow \gamma X(3872)$ [15].

\section{Charmonium states: $\eta_{c}(1 S), \eta_{c}(2 S)$ and $h_{c}$ in the $\psi(2 S)$ decays}

The mass and width of the lowest lying charmonium state, the $\eta_{c}(1 S)$, continue to have large uncertainties when compared to those of other charmonium states [16]. The most recent study by the CLEO-c experiment, using both $\psi(2 S) \rightarrow \gamma \eta_{c}$ and $J / \psi \rightarrow \gamma \eta_{c}$, pointed out a distortion of the $\eta_{c}$ line shape in $\psi(2 S)$ decays [17]. CLEO-c attributed the $\eta_{c}$ line-shape distortion to the energy dependence of the "hindered" M1 transition matrix element.

Based on the data sample of $106 \mathrm{M} \psi(2 S)$ decay events, the $\eta_{c}$ mass and width are measured from the radiative transition $\psi(2 S) \rightarrow \gamma \eta_{c}$ [18]. The $\eta_{c}$ candidates are reconstructed from six exclusive decay modes: $K_{S} K \pi, K^{+} K^{-} \pi^{0}, \eta \pi^{+} \pi^{-}, K_{S} K^{+} \pi^{-} \pi^{+} \pi^{-}, K^{+} K^{-} \pi^{+} \pi^{-} \pi^{0}$, and $3\left(\pi^{+} \pi^{-}\right)$, where $K_{S}$ is reconstructed in $\pi^{+} \pi^{-}$mode, $\eta$ and $\pi^{0}$ from $\gamma \gamma$ final states. For a hindered M1 transition the matrix element acquires terms proportional to $E_{\gamma}^{2}$, which, when combined with the usual $E_{\gamma}^{3}$ term for the allowed transitions, lead to contributions in the radiative width proportional to $E_{\gamma}^{7}$ [19]. Thus, the $\eta_{c}$ lineshape is described by a BW modified by $E_{\gamma}^{7}$ convoluted with a resolution function. It is important to point out that the interference between $\eta_{c}$ and non-resonance in the signal region is also considered. The statistical significance of the interference is $15 \sigma$. This affects the $\eta_{c}$ resonant parameters significantly. Assuming an universal relative phase between the two amplitudes, we obtain $\eta_{c}$ mass and width, $M=2984.2 \pm 0.6 \pm 0.5 \mathrm{MeV} / c^{2}$ and $\Gamma=31.4 \pm 1.2 \pm 0.6 \mathrm{MeV} / \mathrm{c}^{2}$, respectively. Figure 4 shows the fit results in the six $\eta_{c}$ decay modes. With precise measurement of the $\eta_{c}$ mass, one can obtain the hyperfine splitting, $\Delta M_{h f}(1 S)_{c \bar{c}} \equiv M(J / \psi)-M\left(\eta_{c}\right)=112.5 \pm 0.8 \mathrm{MeV} / \mathrm{c}^{2}$, which agrees with the quark model prediction and lattice computations [20,21], and will be helpful for understanding the spin-dependent interactions in hidden quarkonium states.

Using 106 million $\psi(2 S)$ events, BESIII searches for $\eta_{c}(2 S)$ in the decay $\psi(2 S) \rightarrow \gamma \eta_{c}(2 S)$ with $\eta_{c}(2 S) \rightarrow$ $K \bar{K} \pi$ [22]. Figure 5 shows the invariant-mass distributions of $K_{S} K^{+} \pi^{-}$(top) and $K^{+} K^{-} \pi^{0}$ (bottom), where a threeconstraints kinematic fit has been applied (in which the energy of the photon is allowed to float). The solid curve in Fig. 5 shows fitting results of an unbinned maximum likelihood fit with four components: signal, $\chi_{c 1}, \chi_{c 2}$ and other background (coming from $\psi(2 S)$ decays to $\pi^{0} K \bar{K} \pi, K \bar{K} \pi$ and ISR/FSR production of $\left.K \bar{K} \pi \gamma_{I S R} / \gamma_{F S R}\right)$. The fit yields $81 \pm 14$ signal events for the $K_{S} K^{+} \pi^{-}$channel and $46 \pm 11$ for the $K^{+} K^{-} \pi^{0}$ channel, and gives the mass $M\left(\eta_{c}(2 S)\right)=$ $3637.6 \pm 2.9 \pm 1.6 \mathrm{MeV} / \mathrm{c}^{2}$ and width $\Gamma\left(\eta_{c}(2 S)\right)=16.9 \pm$ $6.4 \pm 4.8 \mathrm{MeV} / \mathrm{c}^{2}$. The statistical significance of the signal is more than $11 \sigma$. The product branching fraction $\left(\mathcal{B}\left(\psi(2 S) \rightarrow \gamma \eta_{c}(2 S)\right) \times \mathcal{B}\left(\eta_{c}(2 S) \rightarrow K \bar{K} \pi\right)\right.$ is equal to $\left.1.30 \pm 0.20_{\text {stat }} \pm 0.30_{\text {sys }}\right) \times 10^{-5}$. Combining our result with the BaBar measurement of $\mathcal{B}\left(\eta_{c}(2 S) \rightarrow K \bar{K} \pi\right)$ [23], we find the branching fraction of the M1 transition to be $\mathcal{B}\left(\psi(2 S) \rightarrow \gamma \eta_{c}(2 S)\right)=\left(6.8 \pm 1.1_{\text {stat }} \pm 4.5_{\text {sys }}\right) \times 10^{-4}$. This result is consistent with CLEO-c upper limit [24] and predictions of potential models [25].

We also performed a search for the M1 radiative transition $\psi(2 s) \rightarrow \gamma \eta_{c}(2 S)$ by reconstructing the exclusive $\eta_{c}(2 S) \rightarrow K_{S} K^{+} \pi^{+} \pi^{-} \pi^{-}$decay [26]. An evident $\eta_{c}(2 S)$ peak is observed in $M_{K_{S} K^{+} \pi^{+} \pi^{-} \pi^{-}}$with a statistical significance greater than $4 \sigma$ and the mass and width of $\eta_{c}(2 S)$ are measured to be $M=3646.9 \pm 1.6($ stat $) \pm 3.6($ syst $) \mathrm{MeV} / \mathrm{c}^{2}$ 

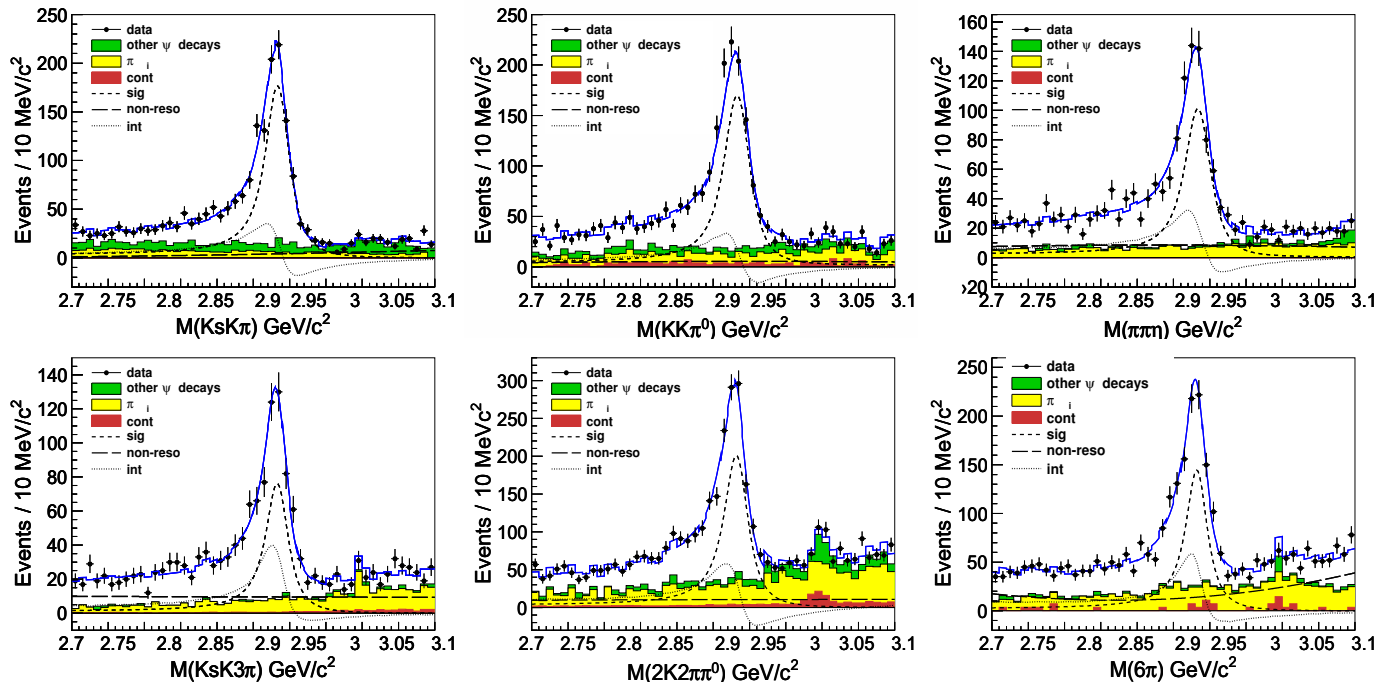

Figure 4. The $M\left(X_{i}\right)$ invariant mass distributions for the decays $K_{S} K^{+} \pi^{-}, K^{+} K^{-} \pi^{0}, \eta \pi^{+} \pi^{-}, K_{S} K^{+} \pi^{+} \pi^{-} \pi^{-}, K^{+} K^{-} \pi^{+} \pi^{-} \pi^{0}$ and $3\left(\pi^{+} \pi^{-}\right)$, respectively, with the fit results superimposed. Points are data and the solid lines are the total fit results [18].
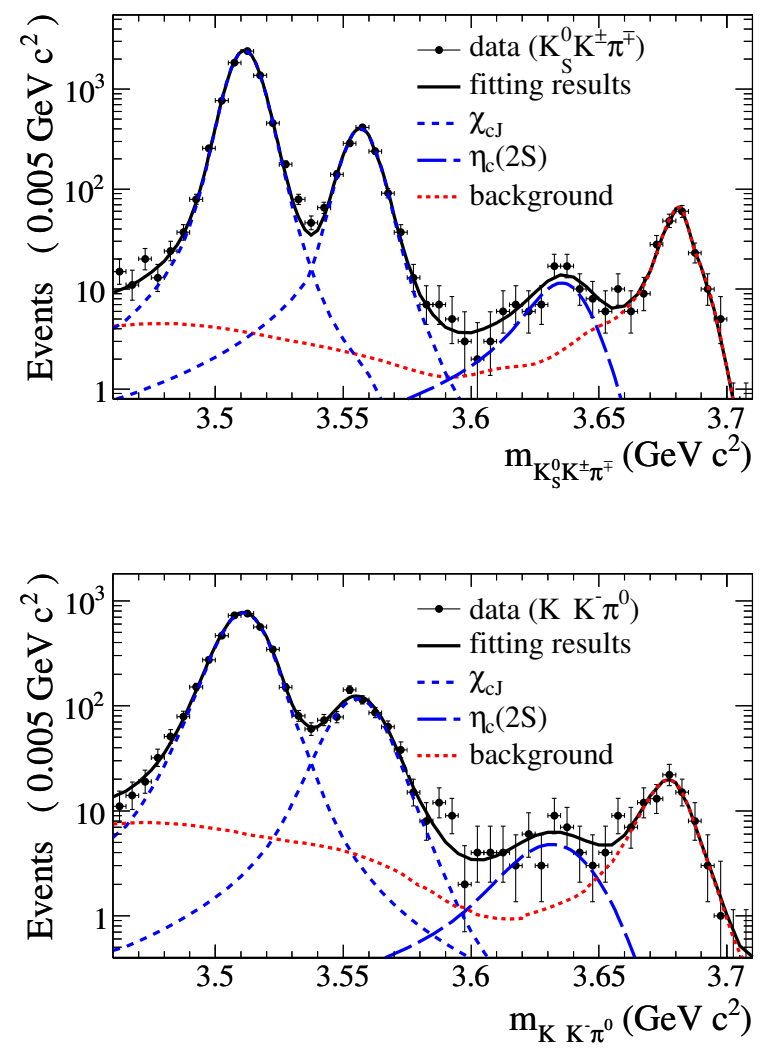

Figure 5. The invariant-mass spectrum for $K_{s} K^{+} \pi^{-}$(top panel), $K^{+} K^{-} \pi^{0}$ (bottom panel), and the simultaneous likelihood fit to the three resonances and combined background sources [22]. and $\Gamma=9.9 \pm 4.8($ stat $) \pm 2.9($ syst $) \mathrm{MeV} / \mathrm{c}^{2}$, which are consistent with the previous BESIII measurements [22].

The BESIII experiment reported the results on the production and decay of the $h_{c}$ using $106 \mathrm{M}$ of $\psi(2 S)$ decay events in 2010 [27], where we studied the distributions of mass recoiling against a detected $\pi^{0}$ to measure $\psi^{\prime} \rightarrow \pi^{0} h_{c}$ both inclusively (E1-untagged) and in events tagged as $h_{c} \rightarrow \gamma \eta_{c}$ (E1-tagged) by detection of the E1 transition photon. In 2011, 16 specific decay modes of $\eta_{c}$ were used to reconstruct $\eta_{c}$ candidates in the decay mode of $h_{c} \rightarrow \gamma \eta_{c}$ [28]. Figure 6 shows the $\pi^{0}$ recoiling mass for the sum of the $16 \eta_{c}$ decay modes in the decay chain of $\psi^{\prime} \rightarrow \pi^{0} h_{c}, h_{c} \rightarrow \gamma \eta_{c}$. We fit the $16 \pi^{0}$ recoil-mass spectra simultaneously that yields $M\left(h_{c}\right)=3525.31 \pm 0.11($ stat. $) \pm 0.14$ (syst. $) \mathrm{MeV} / c^{2}$ and $\Gamma\left(h_{c}\right)=0.70 \pm 0.28$ (stat. $) \pm 0.22$ (syst. $) \mathrm{MeV} / c^{2}$. These results are consistent with the previous BESIII inclusive results and CLEO-c exclusive results.

The centroid of the ${ }^{3} P_{J}$ states $\left(\chi_{c 0}, \chi_{c 1}, \chi_{c 2}\right)$ is known to be $\left\langle M\left({ }^{3} P_{J}\right)\right\rangle=\left[5 M\left({ }^{3} P_{2}\right)+3 M\left({ }^{3} P_{1}\right)+M\left({ }^{3} P_{0}\right)\right]=$ $3525.30 \pm 0.04 \mathrm{MeV} / \mathrm{c}^{2}$ [16]. If the ${ }^{3} P_{J}$ states centroid mass $\left\langle M\left({ }^{3} P_{J}\right)\right\rangle$ is identified as the mass of $M\left({ }^{3} P\right)$, then BESIII observes the hyperfine splitting as $\Delta M_{h f}(1 P)_{c c}=$ $-0.01 \pm 0.11$ (stat. $) \pm 0.15$ (syst.) $\mathrm{MeV} / \mathrm{c}^{2}$ which agrees with zero.

\section{Light hadron physics}

The BESIII is an idea Lab to study the light hadron spectroscopy in the radiative or hadronic $J / \psi / \psi(2 S)$ decays since both $J / \psi$ and $\psi(2 S)$ decays are OZI suppressed. With huge $J / \psi$ and $\psi(2 S)$ samples, we presented recent results, including the partial wave analyses (PWA) of $J / \psi \rightarrow$ $\gamma p \bar{p}, \gamma \eta \eta, \gamma \omega \phi$, and $\psi(2 S) \rightarrow p \bar{p} \pi^{0}$. We have to mention here that both $\eta$ and $\eta^{\prime}$ mesons can be probed in the $J / \psi$ and $\psi(2 S)$ two body decays involving the $\eta / \eta^{\prime}$ meson in the final states. It will be highlight for the BESIII to make 


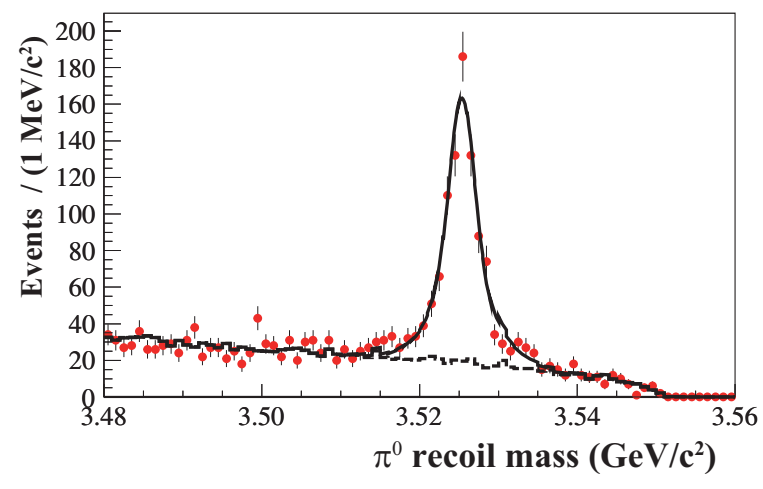

Figure 6. The $\pi^{0}$ recoiling mass for the sum of the $16 \eta_{c}$ decay modes [28].

precision measurements and to search for rare decays of $\eta$ and $\eta^{\prime}$ decays [29].

\subsection{PWA of $J / \psi \rightarrow \gamma p \bar{p}, \gamma \eta \eta, \gamma \omega \phi$, and $\psi(2 S) \rightarrow p \bar{p} \pi^{0}$}

For $J / \psi \rightarrow \gamma p \bar{p}$ decay, the $p \bar{p}$ invariant mass distribution is shown in Fig. 7, where strong $p \bar{p}$ mass threshold enhancement, which denotes as $X(p \bar{p})$, is clearly observed [30]. To determine its spin, parity, mass, width and production rate with high precision, a full PWA with $M_{p \bar{p}}<2.2 \mathrm{GeV} / c^{2}$ was performed after taking into account the final state interactions using the Julich formulation [31]. In the PWA fit the $p \bar{p}$ threshold enhancement, $f_{2}(1910)$ and $f_{0}(2100)$ are described by BreitWigner propagators, and the parameters of the $f_{2}(1910)$ and $f_{0}(2100)$ are fixed at PDG values. Figure 7 shows comparisons of the mass and angular distributions between the data and the PWA fit projections. The mass, width and product BR for the $X(p \bar{p})$ are measured to be: $M=1832_{-5}^{+19}$ (stat. $)_{-17}^{+18}$ (syst.) \pm 19 (model) $\mathrm{MeV} / c^{2}, \Gamma=$ $13 \pm 39$ (stat. $)_{-13}^{+10}$ (syst.) \pm 4 (model) $\mathrm{MeV} / c^{2}$ (a total width of $\Gamma<76 \mathrm{MeV} / c^{2}$ at the $90 \%$ C.L $)$ and $\mathcal{B}(J / \psi \rightarrow \gamma X) \mathcal{B}(X \rightarrow$ $p \bar{p})=\left(9.0_{-1.1}^{+0.4} \text { (stat. }\right)_{-5.0}^{+1.5}$ (syst. $) \pm 2.3($ model $\left.)\right) \times 10^{-5}$, respectively. For the spin-parity determination of the $X(p \bar{p})$, the $0^{-+}$assignment fit is better than that for $0^{++}$or other $J^{P C}$ assignments with statistical significance that are larger than $6.8 \sigma$ [30].

Using 225 million $J / \psi$ events, a PWA of $J / \psi \rightarrow \gamma \eta \eta$ has been performed [32], and the results are summarized in Table 1. The scalar contributions are mainly from $f_{0}(1500), f_{0}(1710)$ and $f_{0}(2100)$, while no evident contributions from $f_{0}(1370)$ and $f_{0}(1790)$ are seen. Recently, the production rate of the pure gauge scalar glueball in $J / \psi$ radiative decays predicted by the lattice QCD [33] was found to be compatible with the production rate of $J / \psi$ radiative decays to $f_{0}(1710)$; this suggests that $f_{0}(1710)$ has a larger overlap with the glueball compared to other glueball candidates (eg. $\left.f_{0}(1500)\right)$. In this analysis, the production rate of $f_{0}(1710)$ and $f_{0}(2100)$ are both about one order of
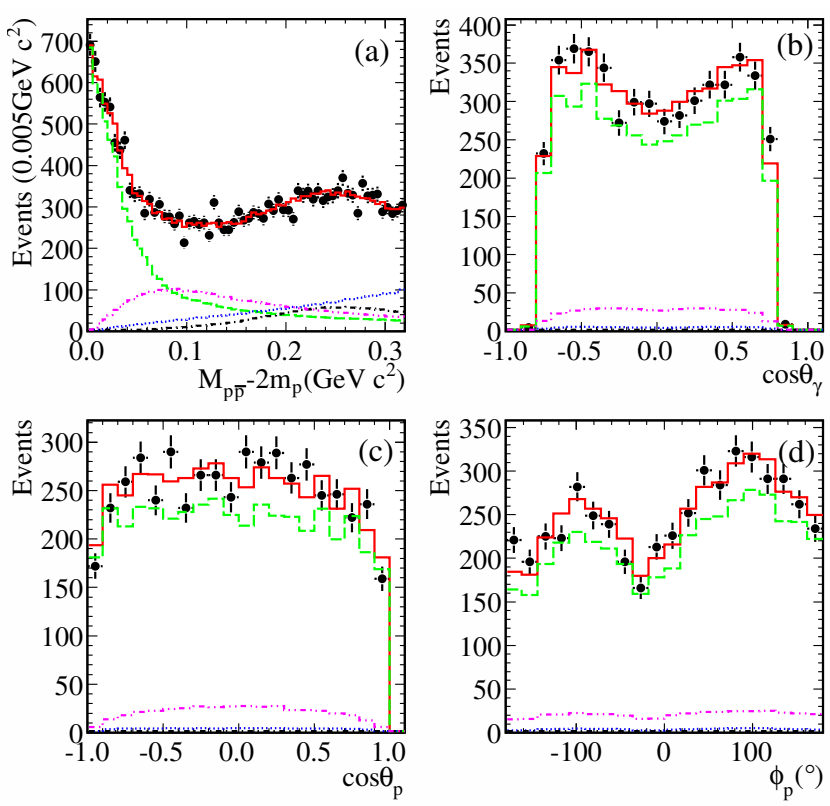

Figure 7. Comparisons between data and PWA fit projection: (a) the $p \bar{p}$ invariant mass; (b)-(d) the polar angle $\theta_{\gamma}$ of the radiative photon in the $J / \psi$ center of mass system, the polar angle $\theta_{p}$ and the azimuthal angle $\phi_{p}$ of the proton in the $p \bar{p}$ center of mass system with $M_{p \bar{p}}-2 m_{p}<50 \mathrm{MeV} / c^{2}$, respectively. Here, the black dots with error bars are data, the solid histograms show the PWA total projection, and the dashed, dotted, dash-dotted and dash-dot-dotted lines show the contributions of the $X(p \bar{p}), 0^{++}$ phase space, $f_{0}(2100)$ and $f_{2}(1910)$, respectively [30].

magnitude larger than that of the $f_{0}(1500)$ and no clear evidence is found for $f_{0}(1370)$, which are both consistent with, at least not contrary to, lattice QCD predictions.

The tensor components, which are dominantly from $f_{2}^{\prime}(1525), f_{2}(1810)$ and $f_{2}(2340)$, also have a large contribution in $J / \psi \rightarrow \gamma \eta \eta$ decays. The significant contribution from $f_{2}^{\prime}(1525)$ is shown as a clear peak in the $\eta \eta$ mass spectrum; a tensor component exists in the mass region from $1.8 \mathrm{GeV} / c^{2}$ to $2 \mathrm{GeV} / c^{2}$, although we cannot distinguish $f_{2}(1810)$ from $f_{2}(1910)$ or $f_{2}(1950)$; and the PWA requires a strong contribution from $f_{2}(2340)$, although the possibility of $f_{2}(2300)$ cannot be ruled out. For the narrow $f_{J}(2220)$, no evident peak is observed in the $\eta \eta$ mass spectrum.

A study of the doubly OZI suppressed decays of $J / \psi \rightarrow \gamma \omega \phi$ is performed [34], and a strong deviation $(>30 \sigma)$ from three-body $J / \psi \rightarrow \gamma \omega \phi$ phase space is observed near the $\omega \phi$ mass threshold, which is consistent with the previous observation reported by the BESII experiment. A PWA with a tensor covariant amplitude that assumes that the enhancement is due to the presence of a resonance, the $X(1810)$, is performed, and confirms that the spin-parity of the $X(1810)$ is $0^{++}$. The mass and width of the $X(1810)$ are determined to be $M=1795 \pm 7(\text { stat })_{-5}^{+13}$ (syst) $\pm 19(\bmod ) \mathrm{MeV} / \mathrm{c}^{2}$ and $\Gamma=95 \pm 10(\text { stat })_{-34}^{+21}($ syst $) \pm 75(\mathrm{mod}) \mathrm{MeV} / \mathrm{c}^{2}$, respectively, and the product branching fraction is measured to 
Table 1. Summary of the PWA results for the $J / \psi \rightarrow \gamma \eta \eta[32]$.

\begin{tabular}{|c|c|c|c|c|}
\hline Resonance & $\overline{\operatorname{Mass}\left(\mathrm{MeV} / \mathrm{c}^{2}\right)}$ & $\overline{\text { Width}\left(\mathrm{MeV} / \mathrm{c}^{2}\right)}$ & $\overline{\mathcal{B}(J / \psi \rightarrow \gamma X \rightarrow \gamma \eta \eta)}$ & Significance \\
\hline$f_{0}(1500)$ & $1468_{-15-74}^{+14+23}$ & $136_{-26-100}^{+41+28}$ & $\left(1.65_{-0.31-1.40}^{+0.26+0.51}\right) \times 10^{-5}$ & $8.2 \sigma$ \\
\hline$f_{0}(1710)$ & $1759 \pm 6_{-25}^{+14}$ & $172 \pm 10_{-16}^{+32}$ & $\left(2.35_{-0.11-0.74}^{+0.13+24}\right) \times 10^{-4}$ & $25.0 \sigma$ \\
\hline$f_{0}(2100)$ & $2081 \pm 13_{-36}^{+24}$ & $273_{-24-23}^{+27+10}$ & $\left(1.13_{-0.10-0.28}^{+0.09+0.64}\right) \times 10^{-4}$ & $13.9 \sigma$ \\
\hline$f_{2}^{\prime}(1525)$ & $1513 \pm 5_{-10}^{+4}$ & $75_{-10-8}^{+12+16}$ & $\left(3.42_{-0.51-1.30}^{+0.43+1.37}\right) \times 10^{-5}$ & $11.0 \sigma$ \\
\hline$f_{2}(1810)$ & $1822_{-24-57}^{+29+66}$ & $229_{-42-155}^{+52+88}$ & $\left(5.40_{-0.67-2.35}^{+0.60+3.42}\right) \times 10^{-5}$ & $6.4 \sigma$ \\
\hline$f_{2}(2340)$ & $2362_{-30-63}^{+31+140}$ & $334_{-54-100}^{+62+165}$ & $\left(5.60_{-0.65-2.07}^{+0.62+2.37}\right) \times 10^{-5}$ & $7.6 \sigma$ \\
\hline
\end{tabular}

be $\mathcal{B}(J / \psi \rightarrow \gamma X(1810)) \times \mathcal{B}(X(1810) \rightarrow \omega \phi)=(2.00 \pm$ $0.08(\text { stat })_{-1.00}^{+0.45}$ (syst $) \pm 1.30($ mod $\left.)\right) \times 10^{-4}$.

To study the baryon spectroscopy, a PWA of $\psi(2 S) \rightarrow$ $p \bar{p} \pi^{0}$ is performed [35]. The branching fraction of this channel has been determined to be $\mathcal{B}\left(\psi(2 S) \rightarrow p \bar{p} \pi^{0}\right)=$ $(1.65 \pm 0.03 \pm 0.15) \times 10^{-4}$. In this decay, $7 N^{*}$ (excited baryons) intermediate resonances are observed. Among these $N^{*}$ resonances, two new resonances are significant, one $(1 / 2)^{+}$resonance with a mass of $2300_{-30-0}^{+40+109} \mathrm{MeV} / \mathrm{c}^{2}$ and width of $340_{-30-58}^{+30+110} \mathrm{MeV} / \mathrm{c}^{2}$, and one $(5 / 2)^{-}$resonance with a mass of $2570_{-10-10}^{+19+34} \mathrm{MeV} / \mathrm{c}^{2}$ and width of $250_{-24-21}^{+14+69}$ $\mathrm{MeV} / \mathrm{c}^{2}$. For the remaining $5 N^{*}$ intermediate resonances, the analysis yields mass and width values which are consistent with those from established resonances.

\subsection{Observation of $X(1840)$ in $J / \psi \rightarrow 3\left(\pi^{+} \pi^{-}\right)$ decays}

We studied the decay $J / \psi \rightarrow \gamma 3\left(\pi^{+} \pi^{-}\right)$with a 225.3 million $J / \psi$ event sample [36]. A structure at $1.84 \mathrm{GeV} / \mathrm{c}^{2}$ is observed in the $3\left(\pi^{+} \pi^{-}\right)$mass spectrum with a statistical significance of 7.6 $\sigma$. As shown in Fig. 8 (Top), fitting the structure $X(1840)$ with a modified Breit-Wigner function yields $M=1842.2 \pm 4.2_{-2.6}^{+7.1} \mathrm{MeV} / \mathrm{c}^{2}$ and $\Gamma=83 \pm 14 \pm 11$ $\mathrm{MeV} / \mathrm{c}^{2}$. The product branching fraction is determined to be $\mathcal{B}(J / \psi \rightarrow \gamma X(1840)) \times \mathcal{B}\left(X(1840) \rightarrow 3\left(\pi^{+} \pi^{-}\right)\right)=$ $\left(2.44 \pm 0.36_{-0.74}^{+0.60}\right) \times 10^{-5}$. The comparison to the BESIII results of the masses and widths of the $X(p \bar{p})$ [30], $X(1810)$ [34], $X(1835)$ [37] and $X(1870)$ [38] are displayed in Fig. 8 (Bottom), where the mass of $X(1840)$ is in agreement with those of $X(1835)$ and $X(p \bar{p})$, while its width is significantly different from any of them. Therefore, based on these data, one cannot determine whether $X(1840)$ is a new state or the signal of a $3\left(\pi^{+} \pi^{-}\right)$decay mode of an existing state. Further study, including an amplitude analysis to determine the spin and parity of the $X(1840)$, is needed to establish the relationship between different experimental observations in this mass region and determine the nature of the underlying resonance or resonances.

\section{3 $\eta$ and $\eta^{\prime}$ physics}

Based on the 225.3 million $J / \psi$ events, we also present the recent results on $\eta$ and $\eta^{\prime}$ decays in this talk. First, we made an attempt to search for their invisible and weak decays via $J / \psi \rightarrow \phi \eta$ and $\phi \eta^{\prime}[39,40]$. These two-body de-
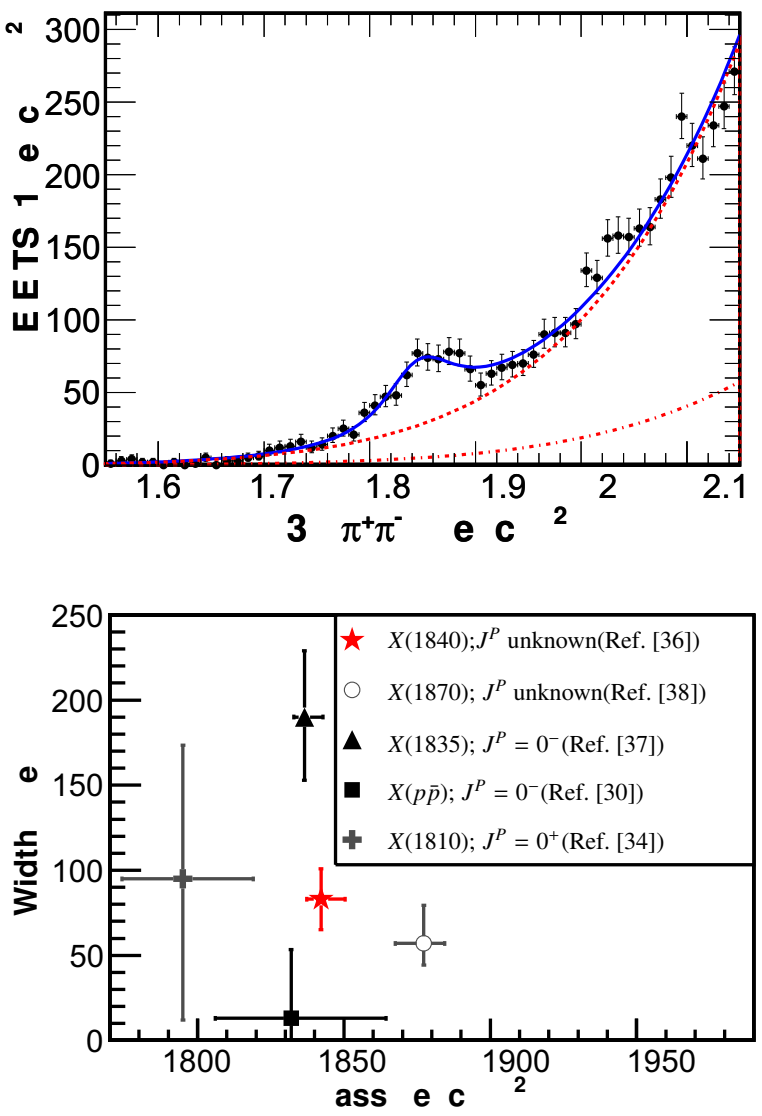

Figure 8. Top: The fit of mass spectrum of $3\left(\pi^{+} \pi^{-}\right)$. The dots with error bars are data; the solid line is the fit result [36]. Bottom: comparisons of observations at BESIII. The error bars include statistical, systematic, and, where applicable, model uncertainties.

cays provide a simple event topology, in which the $\phi$ meson can be reconstructed easily and cleanly with its dominant decay of $\phi \rightarrow K^{+} K^{-}$. Since the $\phi$ and $\eta\left(\eta^{\prime}\right)$ are given with strong boosts in the $J / \psi$ decays, the invisible decays of the $\eta$ and $\eta^{\prime}$ were investigated with the mass spectra recoiling against $\phi$. We find no signal above background for the invisible decays of $\eta$ and $\eta^{\prime}$. To reduce the systematic uncertainty, the upper limits of the ratios, $\frac{\mathcal{B}(\eta \rightarrow \text { invisible })}{\mathcal{B}(\eta \rightarrow \gamma \gamma)}<$ $2.6 \times 10^{-4}$ and $\frac{\mathcal{B}\left(\eta^{\prime} \rightarrow \text { invisible }\right)}{\mathcal{B}\left(\eta^{\prime} \rightarrow \gamma \gamma\right)}<2.4 \times 10^{-2}$, were obtained at the $90 \%$ confidence level [39]. Using the branching frac- 
tion values of $\eta$ and $\eta^{\prime} \rightarrow \gamma \gamma$ from the PDG [16], we determine the invisible decay rates to be $\mathcal{B}(\eta \rightarrow$ invisible $)<$ $1.0 \times 10^{-4}$ and $\mathcal{B}\left(\eta^{\prime} \rightarrow\right.$ invisible $)<5.3 \times 10^{-4}$ at the $90 \%$ confidence level. For the first time a search for the semileptonic weak decay modes $\eta\left(\eta^{\prime}\right) \rightarrow \pi^{+} e^{-} \bar{v}_{e}$ was performed and no signal was observed. The upper limits at the $90 \%$ C.L. are $7.3 \times 10^{-4}$ and $5.0 \times 10^{-4}$ for the ratios of semileptonic over hadronic decay modes $\frac{\mathcal{B}\left(\eta \rightarrow \pi^{+} e^{-} \bar{v}_{e}+c . c .\right)}{\mathcal{B}\left(\eta \rightarrow \pi^{+} \pi^{-} \pi^{0}\right)}$ and $\frac{\mathcal{B}\left(\eta^{\prime} \rightarrow \pi^{+} e^{-} \bar{v}_{e}+c . c .\right)}{\mathcal{B}\left(\eta^{\prime} \rightarrow \pi^{+} \pi^{-} \eta\right)}$ [40], respectively. Using the hadronic branching fraction values of $\eta \rightarrow \pi^{+} \pi^{-} \pi^{0}$ and $\eta^{\prime} \rightarrow \pi^{+} \pi^{-} \eta$ as listed by PDG [16], we determine the semileptonic decay rates to be $\mathcal{B}\left(\eta \rightarrow \pi^{+} e^{-} \bar{v}_{e}+\right.$ c.c. $)<1.7 \times 10^{-4}$ and $\mathcal{B}\left(\eta^{\prime} \rightarrow \pi^{+} e^{-} \bar{v}_{e}+\right.$ c.c. $)<2.2 \times 10^{-4}$ at the $90 \%$ C.L.. The decays of $\eta^{\prime} \pi^{+} \pi^{-} e^{+} e^{-}$and $\eta^{\prime} \rightarrow \pi^{+} \pi^{-} \mu^{+} \mu^{-}$were also studied via $J / \psi \rightarrow \gamma \eta^{\prime}$ [41]. A clear $\eta^{\prime}$ peak is observed in the $M_{\pi^{+} \pi^{-}} e^{+} e^{-}$mass spectrum, and the branching fraction is measured to be $\mathcal{B}\left(\eta^{\prime} \pi^{+} \pi^{-} e^{+} e^{-}\right)=(2.11 \pm 0.12 \pm$ $0.14) \times 10^{-3}$, and no $\eta^{\prime} \rightarrow \pi^{+} \pi^{-} \mu^{+} \mu^{-}$signal is found in the $M_{\pi^{+} \pi^{-} \mu^{+} \mu^{-}}$mass spectrum, and the upper limit is determined to be $\mathcal{B}\left(\eta^{\prime} \rightarrow \pi^{+} \pi^{-} \mu^{+} \mu^{-}\right)<2.9 \times 10^{-5}$ at the $90 \%$ confidence level. With huge data set at the BESIII, we expect more results on $\eta$ and $\eta^{\prime}$ decays to appear soon.

\section{Potential of charm physics at BESIII}

Many of the measurements related to charm decays have been done by other experiments such as BESII and CLEOc, and many are also accessible to the B-factory experiments. What are BESIII's advantages to running at the open charm threshold $[42,43]$ ?

BESIII will not be able to compete with both BABAR and Belle in statistics on charm physics, especially on the rare and forbidden decays of charm mesons. However, data taken at charm threshold still have powerful advantages over the data at $\Upsilon(4 S)$, which we list here [44]: 1) Charm events produced at threshold are extremely clean; 2) The measurements of absolute branching fraction can be made by using double tag events; 3 ) Signal/Background is optimal at threshold; 4) Neutrino reconstruction is clean; 5) Quantum coherence allow simple [45] and complex [46] methods to measure the neutral $D$ meson mixing parameters and strong phase difference [47], and to check for direct $C P$ violation.

For charm physics at BESIII, the first physics results will be the measurements of the leptonic and semileptonic decays of charm mesons. Measurements of the leptonic decays at BESIII will benefit from the fully tagged $D^{+}$and $D_{S}^{+}$decays available at the $\psi(3770)$ and at $\sqrt{s} \sim 4170$ $\mathrm{MeV} / \mathrm{c}^{2}$ or $\sim 4017 \mathrm{MeV} / \mathrm{c}^{2}$ [48]. The leptonic decay rates for $D^{+}$and $D_{S}^{+}$has been measured with a precision of $4.3 \%$ and $2.0 \%$ with the final data from CLEOc. It should be noted that the $D^{+} \rightarrow \tau^{+} v$ decay is reported by CLEOc with an upper limit of $1.2 \times 10^{-3}$ at $90 \%$ C.L. [49]. At BESIII, with 4 times (about $3.0 \mathrm{fb}^{-1}$ ) of the CLEO-c's luminosity, significant gains on these measurements will be made if the systematic errors remain the same. This will allow the validation of theoretical calculations of the decay constants at the $1-2 \%$ level. The neutral D mixing and $C P$ violation in charm sector using quantum correlation are all statistics-starved at CLEO-c, improvement will be made at BESIII experiment.
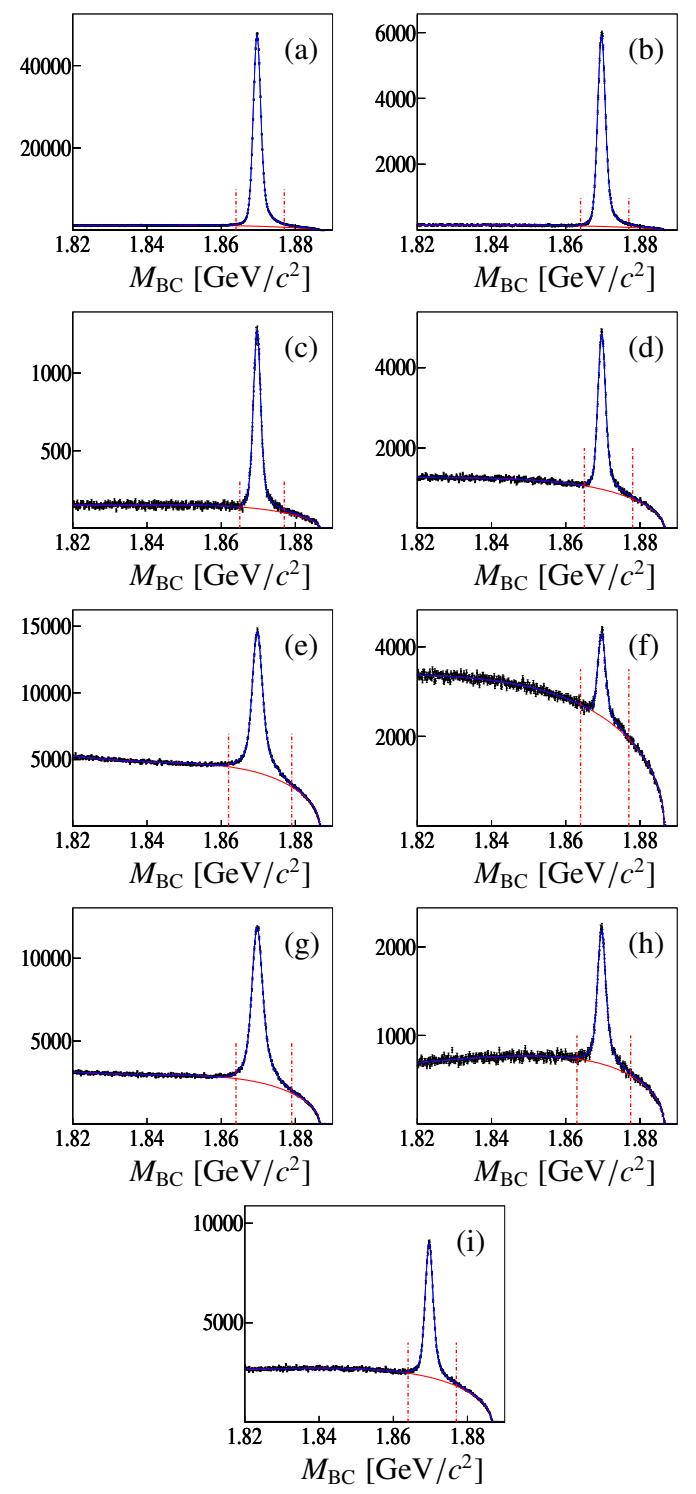

Figure 9. The beam-energy-constrained mass distributions for the different tagged mode combinations, where (a), (b), (c), (d), (e), (f), (g), (h) and (i) are for the modes of $D^{-} \rightarrow K^{+} \pi^{-} \pi^{-}, K_{S}^{0} \pi^{-}$, $K_{S}^{0} K^{-}, K^{+} K^{-} \pi^{-}, K^{+} \pi^{-} \pi^{-} \pi^{0}, \pi^{+} \pi^{-} \pi^{-}, K_{S}^{0} \pi^{-} \pi^{0}, K^{+} \pi^{-} \pi^{-} \pi^{-} \pi^{+}$, and $K_{S}^{0} \pi^{-} \pi^{-} \pi^{+}$, respectively; the two vertical dashed red lines show the tagged $D^{-}$mass region [50].

\subsection{Purely leptonic $D$ decay}

With a sample of $2.9 \mathrm{fb}^{-1}$ taken at open-charm threshold, BESIII experiment measures the decay branching fraction for $D^{+} \rightarrow \mu^{+} v_{\mu}$ and extracts decay constant $f_{D^{+}}$[50]. Decay constant characterizes the strong-interaction physics at the quark-annihilation vertex. In a fully leptonic decay, it parameterizes all of our essential theoretical limitations. Decay constant for $B$ meson also appears in the evaluation of box diagrams, and limits theoretical precision in calculating the neutral $B$ meson mixing. Thus, lack of knowledge of the $B^{0}$ and $B_{s}$ decays constants limits the 
usefulness of precise measurements of $B^{0}-\bar{B}^{0}$ and $B_{s}-\bar{B}_{s}$ oscillations. These mixing data are our best source of information on the CKM matrix elements $V_{t d}$ and $V_{t s}$, which are difficult to measure directly in top decay. The leptonic decay of charm meson presents an opportunity to check LQCD results for decay constants against precision measurements.

We call an event a tagged one if it has a fullyreconstructed $D$ hadronic decay. A sample of tagged events has a greatly reduced background and constrained kinematics, both of which aid studies of how the other $D$ in the event decays. One can infer neutrinos from energy and momentum conservation, allowing "full" reconstruction of (semi)leptonic $D$ decays. The typical tag rates per $D$ (not per pair) are roughly $15 \%$ and $10 \%$ for $D^{0}$ and $D^{+}$, respectively. For pure leptonic decay, the single tagged $D^{-}$meson events are reconstructed in nine nonleptonic decay modes of $D^{-} \rightarrow K^{+} \pi^{-} \pi^{-}, K_{s} \pi^{-}, K_{s} K^{-}$, $K^{+} K^{-} \pi^{-}, K^{+} \pi^{-} \pi^{-} \pi^{0}, \pi^{+} \pi^{-} \pi^{-}, K_{s} \pi^{-} \pi^{0}, K^{+} \pi^{-} \pi^{-} \pi^{-} \pi^{+}$, and $K_{s} \pi^{-} \pi^{-} \pi^{+}$. Mass peaks for the nine hadronic tag modes are shown in Fig. 9. A maximum likelihood fit to the mass spectrum yields the number of the single tagged $D^{-}$events for each of the nine modes. The total number of tagged $D^{-}$events are $170305 \pm 3405$ [50].

The chosen signal variable for the $\mu^{+} v$ decay is the calculated square of the missing-mass of any undetected decay products, shown in Fig. 10; this should of course peak at $M_{v}^{2}=0$ for signal events. The power of $D$-tagging is evident in the clean, isolated signal peak. The events that peak near $M_{\text {miss }}^{2} \simeq 0.25 \mathrm{GeV}^{2} / c^{4}$ are primarily from $D^{+} \rightarrow$ $K_{L}^{0} \pi^{+}$decays, where the $K_{L}^{0}$ is undetected. The numbers of the background events from $D^{+} \rightarrow K_{L}^{0} \pi^{+}$and $D^{+} \rightarrow \pi^{+} \pi^{0}$, as well as $D^{+} \rightarrow \tau^{+} v_{\tau}$, are estimated by analyzing Monte Carlo samples that are 10 times larger than the data. The backgrounds from other $D$ decays are corrected considering the difference in the numbers of events from the data and simulated events in the range from 0.15 to 0.60 $\mathrm{GeV}^{2} / \mathrm{c}^{4}$. These studies show that there are $42.0 \pm 2.3$ background events among the $451 \mathrm{D}^{+} \rightarrow \mu^{+} v_{\mu}$ candidates in the signal region indicated in Fig. 10. After subtracting the number of background events, $409.0 \pm 21.2 \pm 2.3$ signal events $\left(N_{\text {sig }}^{\text {net }}\right)$ for $D^{+} \rightarrow \mu^{+} v_{\mu}$ remain, where the first error is statistical and the second is the systematic associated with the uncertainty of the background estimate. The weighted overall efficiency for detecting $D^{+} \rightarrow \mu^{+} v_{\mu}$ decays is determined to be $\epsilon=0.6403 \pm 0.0012$ by analyzing Monte Carlo simulated events for $D^{+} \rightarrow \mu^{+} v_{\mu}$ in each tagged $D^{-}$mode. We obtain the branching fraction to be

$$
B\left(D^{+} \rightarrow \mu^{+} v_{\mu}\right)=(3.71 \pm 0.19 \pm 0.06) \times 10^{-4},
$$

where the first error is statistical and the second systematic. The measured branching fraction is consistent within error with world average of $\mathcal{B}\left(D^{+} \rightarrow \mu^{+} v\right)=(3.82 \pm 0.33) \times$ $10^{-4}$ [16], but has better precision. The decay constant $f_{D^{+}}$ is then obtained by using $1040 \pm 7$ fs as the $D^{+}$lifetime and 0.2252 as $V_{c d}$ [16]. Our result is

$$
f_{D^{+}}=(203.2 \pm 5.3 \pm 1.8)
$$

$\mathrm{MeV} / \mathrm{c}^{2}$, where the first errors are statistical and the second systematic [50].

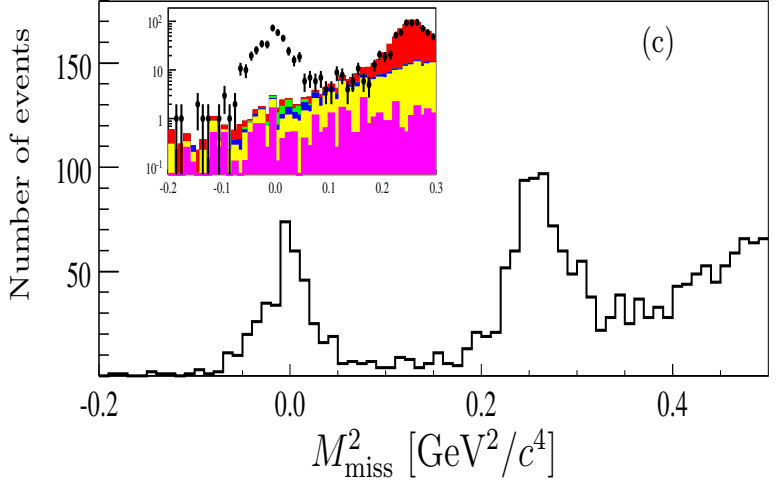

Figure 10. BESIII missing-mass-squared plot for $D^{+} \rightarrow \mu^{+} v$. The insert shows the signal region on a vertical log scale, where dots with error bars are for the data, histograms are sum for the simulated backgrounds from $D^{+} \rightarrow K_{L} \pi^{+}, D^{+} \rightarrow \pi^{+} \pi^{0}, D^{+} \rightarrow$ $\tau^{+} v$ and other decays of $D$ mesons as well as from $e^{+} e^{-} \rightarrow$ non$D \bar{D}$ decays.

\subsection{Semileptonic $D$ decays: $D^{0} \rightarrow K^{-} e^{+} v$ and $\pi^{-} e^{+} v$}

One of the best ways to measure magnitudes of CKM elements is to use semileptonic decays since they are far simpler to understand than hadronic decays and the decay width is $\sim\left|V_{c q}\right|^{2}$. On the other hand, measurements using other techniques have obtained useful values for $V_{c s}$ and $V_{c d}$ [53], and thus semileptonic $D$ decay measurements are a good laboratory for testing theories of QCD. For a $D$ meson decaying into a single hadron $(h)$, the decay rate can be written exactly in terms of the four-momentum transfer defined as:

$$
q^{2}=\left(p_{D}^{\mu}-p_{h}^{\mu}\right)^{2}=m_{D}^{2}+m_{h}^{2}-2 E_{h} m_{D} .
$$

For decays to pseudoscalar mesons and "virtually massless" leptons, the decay width is given by:

$$
\frac{d \Gamma\left(D \rightarrow P e^{+} v\right)}{d q^{2}}=\frac{G_{F}^{2}\left|V_{c q}\right|^{2} p_{P}^{3}}{24 \pi^{3}}\left|f_{+}\left(q^{2}\right)\right|^{2},
$$

where $p_{P}$ is the three-momentum of pseudoscalar meson in the $D$ rest frame, and $f_{+}\left(q^{2}\right)$ is a "form-factor" whose normalization must be calculated theoretically, although its shape can be measured.

The BESIII experiment has taken about $2.9 \mathrm{fb}^{-1}$ data at open-charm threshold during the 2010 and 2011 data runs. Using one-third of the data, a partially-blind analysis has been done with the $D^{0} \rightarrow \mathrm{Kev}$ and $D^{0} \rightarrow \pi e v$ decays. Using the double tag technique, several hadronic $D$ decays are fully reconstructed at first. The following four hadronic $D$ decays are used: $D^{0} \rightarrow K^{-} \pi^{+}, K^{-} \pi^{+} \pi^{0}$, $K^{-} \pi^{+} \pi^{0} \pi^{0}$ and $K^{-} \pi^{+} \pi^{-} \pi^{+}$. After hadronic $D^{0}$ tags are found, we reconstruct signal decay for the other $\bar{D}^{0}$. The signal events with a missing $v$ are inferred using the variable $U=E_{\text {miss }}-\left|P_{\text {miss }}\right|$, similar to missing mass square, where "miss" here refers to the missing energy or momentum. Figure. 11 shows the $U$ distributions and fit projections for the decays of $\bar{D}^{0} \rightarrow K^{+} e^{-} v$ and $\bar{D}^{0} \rightarrow \pi^{+} e^{-} v$. 

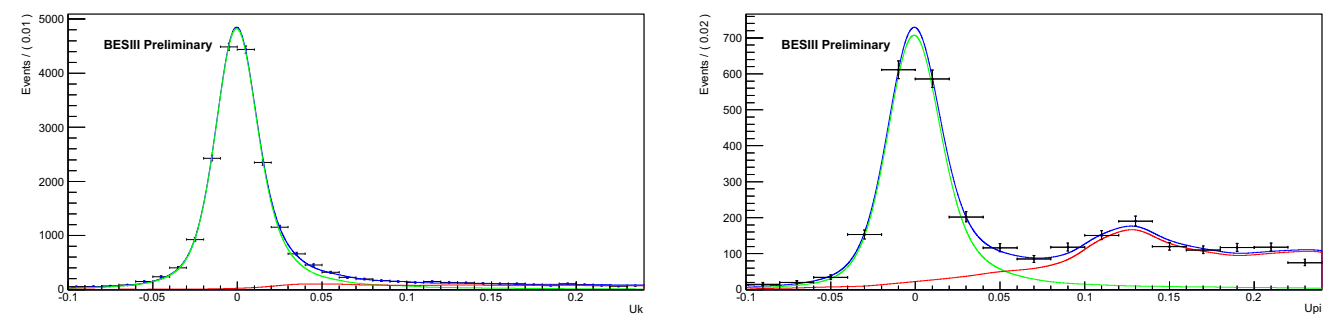

Figure 11. $U$ distributions of $\bar{D}^{0} \rightarrow K^{+} e^{-} v($ left $)$ and $\bar{D}^{0} \rightarrow \pi^{+} e^{-} v($ right $)$.
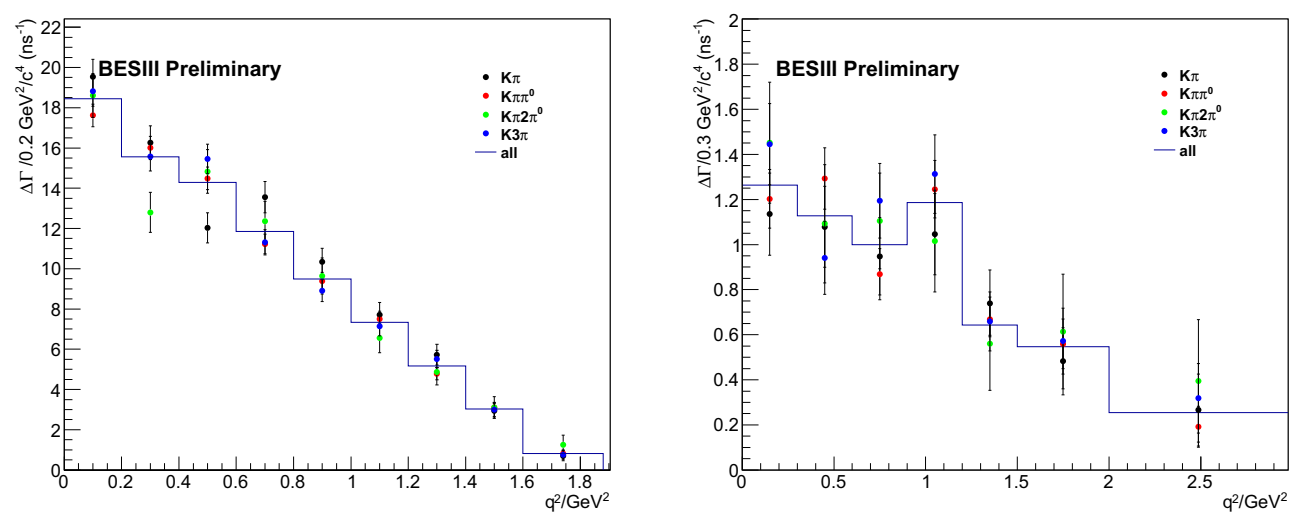

Figure 12. Partial decay rates measurement using individual tag modes (points) and all tag modes combined (histogram) for decay of $\bar{D}^{0} \rightarrow K^{+} e^{-} v$ (left) and $\bar{D}^{0} \rightarrow \pi^{+} e^{-} v$ (right).

Given the signal yields obtained from fitting $U$ distributions and signal efficiencies obtained from signal Monte Carlo, the absolute branching fractions are obtained. Preliminary results of branching fractions are listed in Table 2, and comparisons with results from PDG2012 [16] and CLEO-c results [54] are also made. In order to measure form factor, partial decay rates are measured in different $q^{2}$ bins. $\bar{D}^{0} \rightarrow K^{+} e^{-} v$ candidates are divided into nine $q^{2}$ bins, while $\bar{D}^{0} \rightarrow \pi^{+} e^{-} v$ candidates are divided into seven $q^{2}$ bins. Signal yields in each $q^{2}$ bin are obtained by fitting $U$ distributions in that $q^{2}$ range. Using an efficiency matrix versus $q^{2}$, obtained from Monte-Carlo simulation, and combining with tag yields and tag efficiencies, the partial decay rates are obtained, as shown in Fig. 12.

Table 2. Branching fraction measurement using $923 \mathrm{pb}^{-1}$ of $\psi(3770)$ data from BESIII experiment, and comparisons with results from CLEO-c and PDG2012.

\begin{tabular}{lll}
\hline \hline Experiment & $\mathcal{B}\left(\bar{D}^{0} \rightarrow K^{+} e^{-} v\right)(\%)$ & $\mathcal{B}\left(\bar{D}^{0} \rightarrow \pi^{+} e^{-} v\right)(\%)$ \\
\hline BESIII & $3.542 \pm 0.030 \pm 0.067$ & $0.288 \pm 0.008 \pm 0.005$ \\
CLEO-c & $3.50 \pm 0.03 \pm 0.04$ & $0.288 \pm 0.008 \pm 0.003$ \\
PDG2012 & $3.55 \pm 0.04$ & $0.289 \pm 0.008$ \\
\hline \hline
\end{tabular}

The values of $q^{2}$-dependent form factors in each $q^{2}$ bin can be extracted from the measured partial decay rates. These data can be fitted with different parameterizations of the form factors, and the fit can distinguish between form factor parameterizations. In general, one may express the form factors in terms of a dispersion relation, an approach that has been well established in the literature (see, for example, Ref. [55] and references therein):

$$
\begin{aligned}
f_{+}\left(q^{2}\right)= & \frac{f_{+}(0)}{1-\alpha} \frac{1}{\left(1-q^{2} / m_{\text {pole }}^{2}\right)}+ \\
& \frac{1}{\pi} \int_{\left(m_{D}+m_{P}\right)^{2}}^{\infty} \frac{\operatorname{Im}\left(f_{+}(t)\right)}{t-q^{2}-i \epsilon} d t,
\end{aligned}
$$

where $m_{\text {pole }}$ is the mass of the lowest lying $\left(q_{i} \bar{q}_{f}\right)$ meson with the appropriate quantum numbers: for $D \rightarrow K e v$ it is $D_{s}^{*+}$ and for $D \rightarrow \pi e v$ it is $D^{*+}$, the parameter $\alpha$ gives the relative contribution from the vector pole at $q^{2}=0$, $m_{D}$ is the mass of the $D$ meson, and $m_{P}$ is the mass of the final state pseudoscalar meson. The integral term can be expressed in terms of an infinite series [55]. Typically it takes only a few terms to describe the data. Three different parameterizations of the form factor $f_{+}\left(q^{2}\right)$ are considered. The first parameterization, known as the simple pole model, is dominated by a single pole [56]; the second parameterization is known as the modified pole model [56]; the third parameterization is known as the series expansion [55]. Thus minimized $\chi^{2}$ fits are employed to extract the values of $f_{+}(0)\left|V_{c d(s)}\right|$ using each of the parameterizations. The preliminary results for $f_{+}(0)\left|V_{c d(s)}\right|$ are shown in Table 3 [57]. 
Table 3. Results of $f_{+}(0)\left|V_{c d(s)}\right|$ from individual form factor fits; statistical and systematic uncertainties on the least significant digits are shown in parentheses. Results from CLEO-c are compared.

\begin{tabular}{|c|c|c|}
\hline & \multicolumn{2}{|c|}{$f_{+}(0)\left|V_{c d(s)}\right|$} \\
\hline & BESIII & CLEO-c \\
\hline \multicolumn{3}{|l|}{3 par. Series } \\
\hline $\bar{D}^{0} \rightarrow K^{+} e^{-} v$ & $0.729(8)(7)$ & $0.726(8)(4)$ \\
\hline $\bar{D}^{0} \rightarrow \pi^{+} e^{-} v$ & $0.144(5)(2)$ & $0.152(5)(1)$ \\
\hline \multicolumn{3}{|l|}{2 par. Series } \\
\hline $\bar{D}^{0} \rightarrow K^{+} e^{-} v$ & $0.726(6)(7)$ & $0.717(6)(4)$ \\
\hline $\bar{D}^{0} \rightarrow \pi^{+} e^{-} v$ & $0.140(4)(2)$ & $0.145(4)(1)$ \\
\hline \multicolumn{3}{|l|}{ Modified pole } \\
\hline $\bar{D}^{0} \rightarrow K^{+} e^{-} v$ & $0.725(6)(7)$ & $0.716(6)(4)$ \\
\hline $\bar{D}^{0} \rightarrow \pi^{+} e^{-} v$ & $0.140(3)(2)$ & $0.145(4)(1)$ \\
\hline \multicolumn{3}{|l|}{ Simple pole } \\
\hline $\bar{D}^{0} \rightarrow K^{+} e^{-} v$ & $0.729(5)(7)$ & $0.720(5)(4)$ \\
\hline $\bar{D}^{0} \rightarrow \pi^{+} e^{-} v$ & $0.142(3)(1)$ & $0.146(3)(1)$ \\
\hline
\end{tabular}

In summary for charm physics, the results for the (semi-)leptonic $D$ decays have appeared. However, the BESIII experiment can also measure the strong phase difference between between the doubly Cabibbo-suppressed and and Cabibbo-favored processes, the first preliminary results for $D^{0} \rightarrow K \pi$ were reported [58] recently. We also reported the result on the $D^{+} \rightarrow K_{S} \pi^{+} \pi^{0}$ based on a full amplitude analysis [59]. More analyses on the $D \rightarrow$ $h_{1} h_{2} h_{3}$ Dalitz decays and $D \rightarrow h_{1} h_{2} h_{3} h_{4}$ four-body decays are under going at the BESIII, from which one will extract amplitudes from qusi-two-body contributions. Therefore the strong phase difference, neutral $D$ mixing parameters and $C P$ violation asymmetries can be studied. For the rare charm decays, many decay modes including photons and $\pi^{0}$ in the final states can be probed with high sensitivities (with relative smaller integrated luminosity than that at the $B$ factories), for example, the $D^{0} \rightarrow \gamma \gamma$ decay was reported recently [60].

\section{Summary}

Based on the data samples taken at the BESIII detector from 2008 to 2012, the the recent results on the study of XYZ particles, light hadron spectroscopy, charmonium decays and charm decays are presented, which illustrate the rich physics in the $\mathrm{t}$-charm region. More promising results are expected soon.

\section{References}

[1] M. Ablikim et al. (BESIII Collaboration), Phys. Rev. Lett. 110, 252001 (2013).

[2] Z. Q. Liu et al. (Belle Collaboration), Phys. Rev. Lett. 110, 252002 (2013).

[3] T. Xiao, S. Dobbs, A. Tomaradze and K. K. Seth, arXiv:1304.3036 [hep-ex].
[4] M. Ablikim et al. (BESIII Collaboration), arXiv:1310:4101.

[5] M. Ablikim et al. (BESIII Collaboration), arXiv:1308.2760.

[6] Z. -C. Yang, Z. -F. Sun, J. He, X. Liu and S. -L. Zhu, Chin. Phys. C 36, 6 (2012);

[7] M. Ablikim et al. (BESIII Collaboration), arXiv:1309:1896.

[8] S. K. Choi et al. (Belle Collaboration), Phys. Rev. Lett. 91, 262001 (2003).

[9] D. Acosta et al. (CDF Collaboration), Phys. Rev. Lett. 93, 072001 (2004).

[10] V. M. Abazov et al. (D0 Collaboration), Phys. Rev. Lett. 93, 162002 (2004).

[11] B. Aubert et al. (BaBar Collaboration), Phys. Rev. D 71, 071103 (2005).

[12] A. Abulencia et al. (CDF Collaboration), Phys. Rev. Lett. 98, 132002 (2007).

[13] R. Aaij et al. (LHCb Collaboration), Eur. Phys. J. C 72, 1972 (2012).

[14] A. Abulencia et al. (CDF Collaboration), Phys. Rev. Lett. 96, 102002 (2006).

[15] M. Ablikim et al. (BESIII Collaboration), arXiv:1310:4101.

[16] J. Beringer et al. (Particle Data Group), Phys. Rev. D 86, 010001 (2012).

[17] R. E. Mitchell et al. (CLEO Collaboration), Phys. Rev. Lett. 102, 011801 (2009).

[18] M. Ablikim et al. (BESIII Collaboration), Phys. Rev. Lett. 108, 222002 (2012).

[19] N. Brambilla, Y. Jia and A. Vairo, Phys. Rev. D 73, 054005 (2006).

[20] E. Eichten, K. Gottfried, T. Kinoshita, K. D. Lane and T.-M. Yan, Phys. Rev. D17, 3090 (1978).

[21] Carleton DeTar et al., arXiv:1211.2253 (2012).

[22] M. Ablikim et al. (BESIII Collaboration), Phys. Rev. Lett. 109, 042003 (2012).

[23] B. Aubert et al. (BaBar Collaboration), Phys. Rev. D 78, 012006 (2008).

[24] D. Cronin-Hennessy et al. (CLEO Collaboration), Phys. Rev. D 81, 052002 (2010).

[25] E. J. Eichten, K. Lane and C. Quigg, Phys. Rev. Lett. 89, 162002 (2002).

[26] M. Ablikim et al. (BESIII Collaboration), Phys. Rev. D 87, 052005 (2013).

[27] M. Ablikim et al. (BESIII Collaboration), Phys. Rev. Lett. 104, 132002 (2010).

[28] M. Ablikim et al. (BESIII Collaboration), Phys. Rev. D 86, 092009 (2012).

[29] H. B. Li, J. Phys. G: Nucl. Part. Phys. 36, 085009 (2009).

[30] M. Ablikim et al. (BESIII Collaboration), Phys. Rev. Lett. 108,112003 (2012).

[31] A. Sirbirtsen et al., Phys. Rev. D 71,054010 (2005).

[32] M. Ablikim et al. (BESIII Collaboration), Phys. Rev. D 87, 092009 (2013).

[33] L. C. Gui et al., Phys. Rev. Lett. 110, 021601 (2013). 
[34] M. Ablikim et al. (BESIII Collaboration), Phys.Rev. D 87, 032008 (2013).

[35] M. Ablikim et al. (BESIII Collaboration), Phys. Rev. Lett. 110, 022001 (2013).

[36] M. Ablikim et al. (BESIII Collaboration), Phys. Rev. D 88, 091502 (2013).

[37] M. Ablikim et al. (BESIII Collaboration), Phys. Rev. Lett. 106, 072002 (2011).

[38] M. Ablikim et al. (BESIII Collaboration), Phys. Rev. Lett. 107, 182001 (2011).

[39] M. Ablikim et al. (BESIII Collaboration), Phys. Rev. D 87, 012009 (2013).

[40] M. Ablikim et al. (BESIII Collaboration), Phys. Rev. D 87, 032006 (2013).

[41] M. Ablikim et al. (BESIII Collaboration), Phys. Rev. D 87, 092011 (2013).

[42] H. B. Li, Nucl. Phys. B (Proc. Suppl.) 162, 312 (2006).

[43] H. B. Li, Nucl. Phys. B (Proc. Suppl.) 233,185 (2012).

[44] L. Gibbons, hep-ex/0107079.

[45] M. Gronau, Y. Grossman, and J. L. Rosner, Phys. Lett. B 508,37 (2001).

[46] D. M. Asner and W. M. Sun, Phys. Rev. D73,034024 (2006).

[47] X. D. Cheng, K. L. He, H. B. Li, Y. F. Wang and M. Z. Yang, Phys. Rev. D75, 094019 (2007).

[48] D. Cronin-Hennessy et al. (CLEO Collab.), Phys. Rev. D 80, 072001 (2009).
[49] B. I. Eisenstein et al. (CLEO Collab.),Phys. Rev. D78,052003(2008).

[50] M. Ablikim et al. (BESIII Collaboration), arXiv:1312.0374 [hep-ex].

[51] B. I. Eisenstein et al. (CLEO Collaboration), Phys. Rev. D 78, 052003 (2008).

[52] E. Follana et al. (HPQCD and UKQCD Collaborations), Phys. Rev. Lett. 100,062002 (2008).

[53] M. Artuso, "Status and future perspectives on $V_{c s}$ and $V_{c d}$, Experimental," presented at 4th Int. Workshop on the CKM Unitarity Triangle, Dec., 2006, Nagoya, Japan.

[54] D. Besson et al. (CLEO Collaboration), Phys. Rev. D 80, 032005 (2009)

[55] T. Becher and R. J. Hill, Phys. Lett. B 633, 61 (2006).

[56] D. Becirevic and A. B. Kaidalov, Phys. Lett. B 478, 417 (2000).

[57] C. L. Liu (For BESIII Collaboration), Review of semileptonic charm decays, talk given at CHARM2012.

[58] X. .R. Lu (For BESIII Collaboration), Measurements of strong phase in $D^{0} \rightarrow K \pi$ decay and $y_{C P}$ at BESIII, arXiv:1311.3411 [hep-ex].

[59] M. Ablikim et al. (BESIII Collaboration), arXiv:1401.3083 [hep-ex].

[60] H. Muramatsu (for BESIII Collaboration), Search for $D^{0} \rightarrow \gamma \gamma$ decays, talk given at CHARM2012. 